\title{
RESEARCH
}

Open Access

\section{Epidemiology and clinical management of elbow joint disease in dogs under primary veterinary care in the UK}

\author{
Dan G. O'Neill ${ }^{1 *}$ D, Dave C. Brodbelt ${ }^{1}$, Rebecca Hodge ${ }^{1}$, David B. Church² and Richard L. Meeson²
}

\begin{abstract}
Background: Conditions affecting the elbow joint are a common cause of lameness in dogs. Primary-care veterinary clinical data are now recognised as a valuable research resource. Using data from the VetCompass Programme, this study aimed to report the frequency and risk factors for elbow joint disease in dogs under primary veterinary care in the UK and describe clinical management.
\end{abstract}

Results: From 455,069 dogs under veterinary care, the one-year period prevalence for elbow joint disease diagnosis was $0.56 \%$ (95\% Cl: 0.53-0.60). Of 616 incident cases, the most common specific variants of elbow joint disease were osteoarthritis $(n=468,75.97 \%)$, elbow dysplasia $(190,30.84 \%)$ and traumatic $(41,6.66 \%)$. The most common signs described by the owners were lameness $(n=466,75.65 \%)$, difficulty exercising $(123,19.97 \%)$ and pain $(86,13.96 \%)$. The most common findings recorded on veterinary examination were pain $(n=283,45.94 \%)$, lameness $(278,45.13 \%)$ and reduced range of movement $(243,39.45 \%)$. Common medications used included non-steroidal anti-inflammatory drugs $(n=544,88.31 \%)$, tramadol $(121,19.64 \%)$ and disease modifying agents $(118,19.16 \%)$. Of 109 deaths involving euthanasia with information available from the 616 incident cases, elbow joint disease contributed to the decision to euthanase in 45 (41.28\%) dogs.

Five breeds showed increased odds of elbow joint disease compared with crossbred dogs: Rottweiler (OR: 6.16, 95\% Cl 3.89-9.75), Labrador Retriever (OR: 5.94, 95\% Cl 4.65-7.60), German Shepherd Dog (OR: 4.13, 95\% Cl 2.88-5.93), Golden Retriever (OR: 3.11, 95\% Cl 1.93-5.00) and English Springer Spaniel (OR: 2.00, 95\% Cl 1.26-3.18). Additional risk factors included having an adult bodyweight that was equal or higher than their breed/sex mean, advancing age, being male, being neutered, being insured and larger bodyweight.

Conclusions: Elbow joint disease is a relatively common diagnosis in dogs and has a high welfare impact as evidenced by the high proportion of cases recorded with pain, lameness and analgesic therapy. There are strong breed predispositions, in particular for large breed dogs. These findings present a clear case for improved breeding programmes to reduce the burden of elbow joint disease.

Keywords: General practice, First opinion, VetCompass, Arthritis, Degenerative joint disease, Osteoarthritis, Elbow dysplasia

\footnotetext{
* Correspondence: doneill@rvc.ac.uk

${ }^{1}$ Production and Population Health, The Royal Veterinary College, Hawkshead

Lane, North Mymms, Hatfield, Herts AL9 7TA, UK

Full list of author information is available at the end of the article
}

(c) The Author(s). 2020 Open Access This article is distributed under the terms of the Creative Commons Attribution 4.0 International License (http://creativecommons.org/licenses/by/4.0/), which permits unrestricted use, distribution, and reproduction in any medium, provided you give appropriate credit to the original author(s) and the source, provide a link to the Creative Commons license, and indicate if changes were made. The Creative Commons Public Domain Dedication waiver (http://creativecommons.org/publicdomain/zero/1.0/) applies to the data made available in this article, unless otherwise stated. 


\section{Plain English summary}

The canine elbow can be affected by several different diseases (e.g. elbow dysplasia, osteoarthritis) that cause pain and loss of freedom to exercise. First opinion veterinary clinical records are a valuable research resource that benefit from recording of medical records at the time of the clinical event. This study aimed to report the frequency and risk factors for elbow joint disease and describe clinical management in dogs attending first opinion veterinary practices in the UK.

The VetCompass ${ }^{\mathrm{Tm}}$ Programme shares anonymised clinical records with first-opinion veterinary practices in the UK for research. These records on dogs were searched for cases of elbow joint disease and additional information was extracted on each case. The study included 455,069 dogs at 304 clinics in the UK during 2013. The overall proportion of dogs with elbow joint disease was $0.56 \%$. The average adult bodyweight of cases was $32.45 \mathrm{~kg}$ and the average age at diagnosis was 6.84 years. The most common presenting signs recorded by owners were lameness (75.65\%), difficulty exercising (19.97\%) and pain (13.96\%).

Five breeds had increased odds of elbow joint disease compared with crossbred dogs: Rottweiler $(\times 6.16)$, Labrador Retriever (× 5.94), German Shepherd Dog (x: 4.13), Golden Retriever $(\times 3.11)$ and English Springer Spaniel $(\times 2.00)$. Dogs that were heavier than their breed/sex had 2.00 times the odds of elbow joint disease. Aging, heavier bodyweight, being male, being neutered and being insured were also associated with increased odds.

At least one medication was prescribed for $93.02 \%$ of cases. Common medications used included non-steroidal anti-inflammatory drugs [NSAIDS] (88.31\%), tramadol (19.64\%) and disease modifying agents (19.16\%). Of 109 dogs that were euthanased for any reason during the study period, elbow joint disease contributed to the decision to euthanase in 45 (41.28\%).

This study shows that elbow joint disease is a relatively common diagnosis in dogs and has a high welfare impact. There are strong breed predispositions, in particular for large breed dogs. These findings present a clear case for improved breeding programmes to reduce the burden of elbow joint disease in dogs.

\section{Background}

Conditions affecting the elbow joint are a common cause of lameness in both young [1] and older dogs [2]. The elbow was identified as the most frequently abnormal joint in a study of radiographic abnormalities based on screening of 1018 Labradors over one year of age [3]. The canine elbow can be affected by several different diseases, including elbow dysplasia [4], osteoarthritis, humeral intracondylar fissures $[5,6]$, congenital luxations, soft-tissue problems [7] and septic arthritis [2]. However, each of these diseases usually results in a similar clinical presentation that includes lameness, joint pain and reduced elbow movement, and that adversely affect welfare $[1,7]$. Overall, the syndromic group of diseases known collectively as 'elbow dysplasia' predominates as the most commonly reported group of conditions affecting the elbow of large and giant breed dogs and generally results in secondary osteoarthritis $[1,4]$. The vast majority of literature associated with diseases of the canine elbow focuses on elbow dysplasia and its management, and these studies have generally been based on the referral or disease-screening subsets of the dog population $[4,8]$.

Elbow dysplasia describes a group of conditions that were first described in 1965 as generalised elbow osteoarthrosis with or without ununited anconeal process and which are thought to arise from abnormal growth of the elbow joint [9]. The International Elbow Working Group (IEWG) publishes an annual report on the state of diagnosis and management of elbow dysplasia in dogs. Two decades after their first landmark report, there remains disagreement about exactly which pathologies of the elbow are forms of elbow dysplasia with variable inclusion of ununited medial epicondyle and elbow incongruity $[8,10]$. The undisputed pathologies under this syndromic umbrella term include fragmented medial coronoid process, osteochondrosis of the humeral condyle, and ununited anconeal process $[4,11$, 12]. Although ununited anconeal process and osteochondritis dissecans of the humerus are relatively straightforward to identify on plain radiographs, diagnosis of disease associated with the medial coronoid process (including the aforementioned fragmentation) is problematic due to superimposition of the medial epicondyle and other soft-tissues [11-13]. Therefore, diagnosis is based on associated radiographic secondary changes in the joint (ulna notch sclerosis, new bone formation on the anconeal process or radial head) in the absence of radiographically identifiable lesions, as surrogate marker for medial coronoid disease [14, 15]. However, these changes are only indicative of elbow osteoarthritis [16]. Advanced modalities, such as computed tomography and arthroscopy have significantly increased the accuracy of diagnosis of elbow pathologies such as medial coronoid disease $[17,18]$ but are typically only available in referral clinics and hence only for a small and biased subset of dogs [19].

Elbow dysplasia has a reported prevalence of $17 \%$ in US Labrador Retrievers, and 70\% in Bernese Mountain Dogs from the Netherlands [20]. Although large breed dogs and males are over-represented [21], increased risk has also been reported in some chondrodystrophic breeds such as the French Bulldog and Dachshund [4]. To date, most epidemiological studies of elbow dysplasia, or elbow disease more generally, have been based on 
subsets of animals from treated populations (usually referral cases $[4,8,12])$ or disease screening programmes [22-26]. These approaches, however, fail to account for the impact of the condition in the wider general dog population.

Several large epidemiology studies have reported genetic predisposition to elbow dysplasia in certain dog breeds, in particular Rottweilers, German Shepherd Dogs, Bernese Mountain Dogs and Labrador Retrievers, with estimates of heritability ranging from $0.10-0.38$ [22-25]. When considering specific diseases such as medial coronoid process disease, there is increased heritability such as in the German Shepherd Dog at 0.57 [26], indicating an important genetic contribution to elbow dysplasia. A bimodal pattern of presentation with two age related peaks has been described for elbow dysplasia; young dogs less than or around 4-12 months of age, and then older dogs of around 8 years [1, 4]. However, these data are based on referral populations where biases such as financial factors may have influenced the patterns seen.

Primary-care veterinary clinical data are now recognised as a valuable research resource that benefit from contemporaneous recording of medical records at the time of the clinical event, and from the recording of cohort data over time and at a veterinary level of clinical precision [27, 28]. Such data have been validated for research purposes by several previous reports on diverse conditions in dogs including road traffic accidents [29], appendicular osteoarthritis [30], dystocia [31], urinary incontinence [32], and corneal ulcerative disease [33]. The current study aimed to fill the information gap on the epidemiology of elbow joint disease by estimating the prevalence and incidence of elbow joint disease in dogs attending primary-care veterinary practice in the UK and evaluating breed as a risk factor for incident elbow joint disease. The study also aimed to report summary statistics on diagnostics, management and outcomes that can contribute to benchmarking for clinical audit and governance [34, 35].

Based on the prior but potentially biased information in the literature, it was hypothesized that purebred dogs, in particular Labrador Retriever, Bernese Mountain Dog, Rottweiler German Shepherds, English Springer Spaniels and French Bulldogs, older dogs, male dogs, and heavier dogs would have higher odds of elbow joint disease than crossbred, younger, female and lighter dogs respectively.

\section{Methods}

The VetCompass ${ }^{\mathrm{Tm}}$ Programme collates de-identified electronic patient record (EPR) data from primary-care veterinary practices in the UK for epidemiological research $[27,36]$. VetCompass ${ }^{\text {Tx }}$ collects information fields that include species, breed, date of birth, sex, neuter status, insurance status and bodyweight, and clinical information from free-form text clinical notes and summary diagnosis terms (VeNom codes) [37], plus treatment and deceased status with relevant dates. The EPR data were extracted from practice management systems using integrated clinical queries and uploaded to a secure VetCom$\operatorname{pass}^{\mathrm{Tnx}}$ structured query language database [27].

A cohort study design was used to estimate the prevalence, incidence and risk factors for elbow joint disease [38]. The sampling frame for the current study included dogs under veterinary care within the VetCompass ${ }^{\mathrm{mm}}$ database for a one-year period from January 1st 2013 to December 31st 2013. Dogs 'under veterinary care' were defined as any dog with either at least one EPR recorded from January 1st to December 31st 2013 or, alternatively, at least one EPR both before and after 2013. Sample size calculations estimated that a sample of 149,282 dogs would be required to estimate an incidence risk for a disorder expected to occur in $1.0 \%$ of overall population with a $0.05 \%$ confidence limit assuming a UK population size of 8,000,000 dogs (Epi Info 7 CDC, 2019, Murray et al., 2010). Ethical approval was granted by the RVC Ethics and Welfare Committee (reference number SR2018-1652).

Case definition for an elbow joint disease case required that a final diagnosis of elbow joint disease (or synonym) was recorded in the EPR for a disorder that was present during the 2013 study period. The clinical decisionmaking process used for diagnosis of elbow joint disease was at the discretion of the attending veterinary surgeon. Case-finding involved initial screening of all EPRs for candidate elbow joint disease cases by searching the clinical free-text field and the VeNom term field using the single search term elbow. The candidate cases were randomly ordered and the clinical notes of a subset based on the power calculation estimate were manually reviewed in detail to evaluate for case inclusion. Information was extracted on cases to describe whether these were pre-existing (diagnosed prior to 2013) or incident (first recorded diagnosed during 2013) cases. Additional information extracted on the incident cases included the presenting signs described by the owners, whether the elbow joint disease was an incidental finding during a clinical examination for another presentation, specific type of elbow joint disease, findings recorded on veterinary examination, diagnostic process, medication, surgery and mortality information.

A purebred variable categorised all dogs of recognisable breeds as 'purebred' and the remaining dogs as 'crossbred' [39]. A breed variable included individual breeds represented by over 4000 dogs in the overall study population or with $\geq 7$ incident elbow joint disease cases, a grouped category of all remaining purebreds and a general grouping of crossbred dogs. This approach was 
taken to facilitate statistical power for the individual breed analyses [40]. A Kennel Club breed group variable classified breeds recognised by the UK Kennel Club into their relevant breed groups (Gundog, Hound, Pastoral, Terrier, Toy, Utility and Working) and all remaining types were classified as non-Kennel Club recognised [39]. Sex (female, male, unavailable) and neuter (neutered, entire, unavailable) variables described the status recorded at the final EPR. An insurance variable described whether a dog was insured at any point during the study period. Age (years) was calculated for incident cases at the date of first recorded diagnosis and for all remaining dogs at the final date of the study period (December 31st, 2013). An age variable categorised age (years) into six groups $(<3.0,3.0$ $-<6.0,6.0-<9.0,9.0-<12.0, \geq 12.0$, unavailable). Adult bodyweight described the maximum bodyweight $(\mathrm{kg})$ recorded at any date for dogs $>18$ months old. An adult bodyweight variable categorised adult bodyweight into six groups $(<10.0 \mathrm{~kg}, 10.0-<20.0 \mathrm{~kg}, 20.0-<30.0 \mathrm{~kg}, 30.0$ $-<40.0 \mathrm{~kg}, \geq 40.0 \mathrm{~kg}$, unavailable). A bodyweight relative to breed mean variable characterised the adult bodyweight of individual dogs as either below or equal/above the mean adult bodyweight for their breed and sex within the overall study population. This variable allowed assessment of adult bodyweight effects within each breed/sex combination.

Following data checking and cleaning in Excel (Microsoft Office Excel 2013, Microsoft Corp.), analyses were conducted using Stata Version 13 (Stata Corporation). The one-year period prevalence with $95 \%$ confidence intervals (CI) described the probability of evidence in the clinical records that confirmed the presence of elbow joint disease at any time during 2013. The elbow joint disease cases included both pre-existing (first diagnosed before 2013) and incident (newly diagnosed during 2013) cases. Because the sampling design involved manual verification of a subset of the candidate cases, the predicted case count for 2013 was calculated using the Stata survey function that weighted the verified case numbers by the inverse of the proportion of candidate cases manually confirmed [41]. The CI estimates were derived from standard errors, based on approximation to the binomial distribution [42]. This approach was repeated to similarly report the one-year incidence risk for newly diagnosed elbow joint disease cases during 2013. Descriptive statistics characterised the risk factors separately for the non-case and prevalent case dogs.

Risk factor analysis included only incident elbow joint disease dogs as cases while non-cases included all dogs that were not originally screened as candidate elbow joint disease cases. This focus on incident cases allowed interpretation of results as risk factors for "becoming" a case rather than for "being" a case [43]. Binary logistic regression modelling was used to evaluate univariable associations between risk factors (purebred, breed, Kennel Club breed group, adult bodyweight, bodyweight relative to breed/sex mean, age, sex, neuter and insurance) and elbow joint disease during 2013. Because breed was a factor of primary interest for the study, purebred, and Kennel Club breed group (variables that are highly collinear with breed) and adult bodyweight (a defining characteristic of individual breeds) were excluded from the initial breed multivariable modelling. Instead, each of these variables individually replaced the breed variable in the main final model in order to evaluate their effects after taking account of the other variables. Risk factors with liberal associations in univariable modelling $(P<0.2)$ were taken forward for multivariable evaluation. Model development used manual backwards stepwise elimination. Clinic attended was evaluated as a random effect and pair-wise interaction effects were evaluated for the final model variables [44]. The area under the ROC curve and the Hosmer-Lemeshow test were used to evaluate the quality of the model fit and discrimination (non-random effect model) [44, 45]. Statistical significance was set at $P<0.05$.

\section{Results \\ Demography}

The denominator population comprised 455,069 dogs under veterinary care at 304 clinics in the UK during 2013. Of 12,060 candidate cases identified, 3751 (31.1\%) were manually checked to confirm 804 elbow joint disease cases from this sample. After accounting for the effects of the subsampling protocol, the estimated oneyear period prevalence for elbow joint disease diagnosis in dogs overall was $0.56 \%$ (95\% CI: $0.53-0.60)$. The breeds with the highest elbow joint disease prevalence were Labrador Retriever $(2.54,95 \%$ CI $2.37-2.71)$, Rottweiler (1.99, 95\% CI 1.63-2.40), Golden Retriever (1.47, 95\% CI 1.18-1.83), German Shepherd Dog (1.28, 95\% CI 1.10-1.50), and English Springer Spaniel (0.92, 95\% CI 0.75-1.12) (Fig. 1). There were 616/804 (76.6\%) of the overall cases that were incident in 2013. After accounting for the effects of the subsampling protocol, the estimated one-year incidence risk for elbow joint disease diagnosis was $0.45 \%$ (95\% CI, $0.41-0.48$ ) based on these 616 incident cases.

Of the incident elbow joint disease cases with data available for that variable, $515(83.74 \%)$ were purebred, 244 (39.61\%) were female, $360(74.69 \%)$ were neutered and $182(81.61 \%)$ were insured. Dogs with elbow joint disease had a median adult bodyweight of $32.45 \mathrm{~kg}$ (IQR: 22.00-40.00, range 3.00-88.00) and median age at diagnosis was 6.84 years (IQR: 2.45-9.97, range 0.23-17.00). The age distribution at diagnosis showed a bimodal pattern with a sharp peak in year 1 and a more gradual peak in years 6-10 (Fig. 2). The most common breeds 


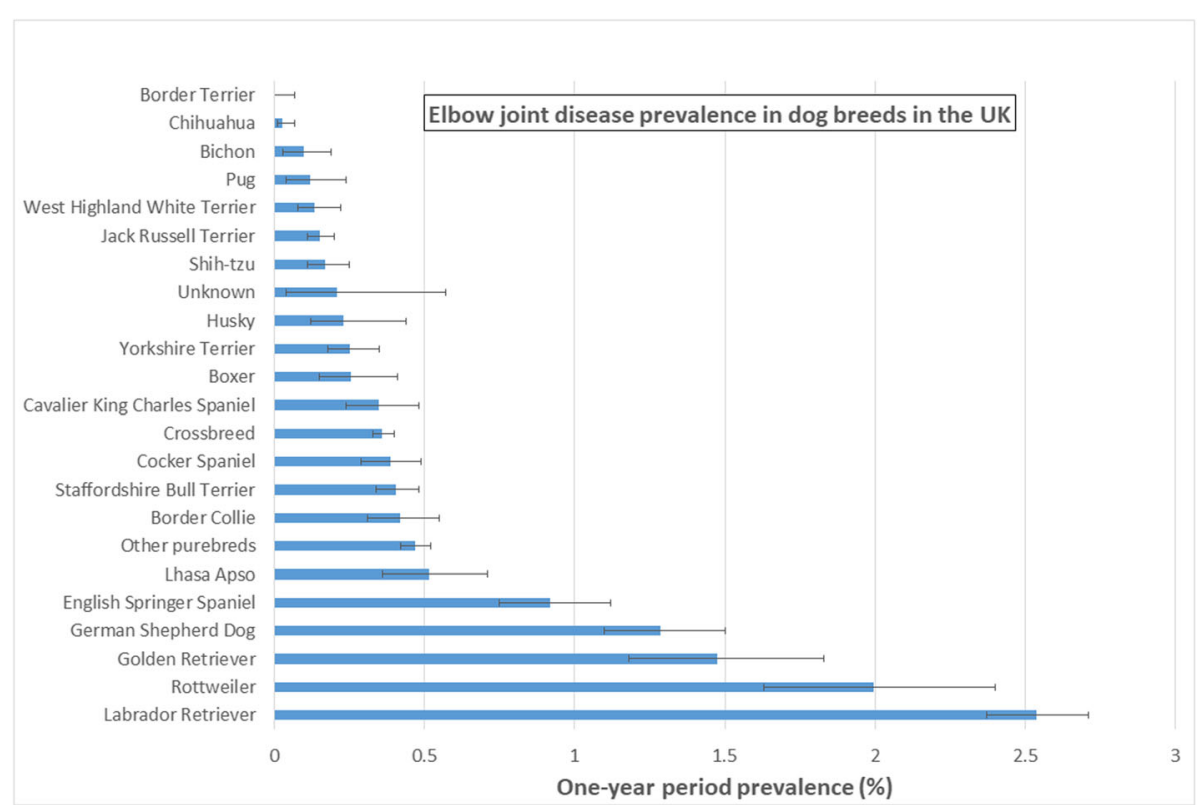

Fig. 1 One-year (2013) period prevalence of elbow joint disease diagnosis in commonly affected dog breeds the VetCompass ${ }^{T M}$ Programme under primary veterinary care in the UK in 2013. The error bars show the 95\% confidence interval. $(n=804)$

among the incident elbow joint disease cases were Labrador Retriever (189, 30.68\%), German Shepherd Dog (43, 6.98\%), Staffordshire Bull Terrier (32, 5.19\%) and Rottweiler (23, 3.73\%), along with crossbred dogs (100, 16.23\%) (Table 1). The median (IQR, count) age at first diagnosis for breeds with over 20 incident cases was:
Labrador Retriever 6.42 years $(2.50-9.08, n=188)$, German Shepherd Dog 5.64 (0.80-7.77, 42), Staffordshire Bull Terrier 8.02 (3.16-10.87, 32), Rottweiler 7.20 (1.478.17, 23), English Springer Spaniel 7.00 (1.77-12.27, 21), Golden Retriever $9.75(5.27-11.65,21)$ and crossbred dogs 7.65 (3.39-10.95, 100).

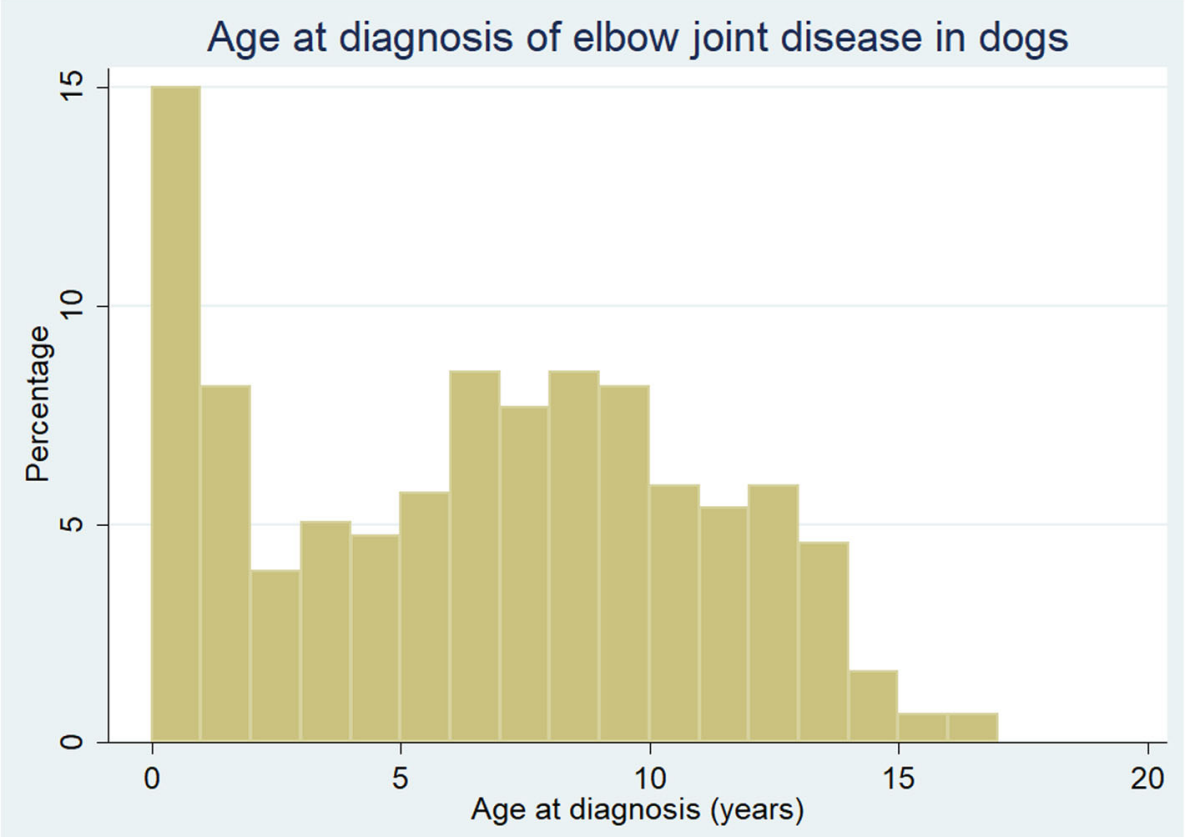

Fig. 2 Distribution of age at first diagnosis of elbow joint disease diagnosis in dogs from the VetCompass ${ }^{\mathrm{TM}}$ Programme under primary veterinary care in the UK in 2013. $(n=616)$ 
Table 1 Descriptive and univariable logistic regression results for risk factors associated with incident diagnoses during 2013 of elbow joint disease in dogs under primary veterinary care in the VetCompass ${ }^{\text {TM }}$ Programme in the UK. Column percentages shown in brackets. ${ }^{*} \mathrm{Cl}$ confidence interval

\begin{tabular}{|c|c|c|c|c|c|c|c|}
\hline Variable & Category & Case No. (\%) & $\begin{array}{l}\text { Non-case No. } \\
(\%)\end{array}$ & $\begin{array}{l}\text { Odds } \\
\text { ratio }\end{array}$ & $95 \% \mathrm{Cl}^{*}$ & $\begin{array}{l}\text { Category } \\
P \text {-value }\end{array}$ & $\begin{array}{l}\text { Variable } \\
P \text {-value }\end{array}$ \\
\hline \multirow[t]{2}{*}{ Purebred status } & Crossbred & $100(16.26)$ & $110,124(24.96)$ & Base & & & $<0.001$ \\
\hline & Purebred & $515(83.74)$ & $331,113(75.04)$ & 1.72 & $1.38-2.12$ & $<0.001$ & \\
\hline \multirow[t]{23}{*}{ Breed } & Crossbreed & $100(16.23)$ & $110,124(24.87)$ & 1.00 & & & $<0.001$ \\
\hline & Labrador Retriever & 189 (30.68) & $30,894(6.98)$ & 6.74 & $5.29-8.59$ & $<0.001$ & \\
\hline & Rottweiler & $23(3.73)$ & 4999 (1.13) & 5.07 & $3.22-7.98$ & $<0.001$ & \\
\hline & Golden Retriever & $21(3.41)$ & $5318(1.20)$ & 4.35 & $2.71-6.97$ & $<0.001$ & \\
\hline & German Shepherd Dog & $43(6.98)$ & $11,848(2.68)$ & 4.00 & $2.79-5.72$ & $<0.001$ & \\
\hline & English Springer Spaniel & $22(3.57)$ & $10,709(2.42)$ & 2.26 & $1.43-3.59$ & 0.001 & \\
\hline & Other purebreds & $100(16.23)$ & $82,952(18.74)$ & 1.33 & $1.01-1.75$ & 0.045 & \\
\hline & Lhasa Apso & $8(1.30)$ & $6773(1.53)$ & 1.30 & $0.63-2.67$ & 0.474 & \\
\hline & Cavalier King Charles Spaniel & $11(1.79)$ & $9924(2.24)$ & 1.22 & $0.65-2.28$ & 0.53 & \\
\hline & Border Collie & $13(2.11)$ & $11,834(2.67)$ & 1.21 & $0.68-2.16$ & 0.519 & \\
\hline & Staffordshire Bull Terrier & $32(5.19)$ & $31,897(7.20)$ & 1.10 & $0.74-1.65$ & 0.624 & \\
\hline & Cocker Spaniel & $14(2.27)$ & $15,458(3.49)$ & 1.00 & $0.57-1.75$ & 0.993 & \\
\hline & Yorkshire Terrier & $11(1.79)$ & $15,296(3.45)$ & 0.79 & $0.42-1.48$ & 0.463 & \\
\hline & Unavailable & $1(0.16)$ & $1502(0.34)$ & 0.73 & $0.10-5.26$ & 0.758 & \\
\hline & Boxer & $4(0.65)$ & $6075(1.37)$ & 0.73 & $0.27-1.97$ & 0.529 & \\
\hline & Pug & $2(0.32)$ & $5318(1.20)$ & 0.41 & $0.10-1.68$ & 0.217 & \\
\hline & Jack Russell Terrier & $10(1.62)$ & $27,407(6.19)$ & 0.40 & $0.21-0.77$ & 0.006 & \\
\hline & West Highland White Terrier & $4(0.65)$ & $11,838(2.67)$ & 0.37 & $0.14-1.01$ & 0.053 & \\
\hline & Shih-tzu & $5(0.81)$ & $14,882(3.36)$ & 0.37 & $0.15-0.91$ & 0.03 & \\
\hline & Husky & $1(0.16)$ & $4070(0.92)$ & 0.27 & $0.04-1.94$ & 0.193 & \\
\hline & Bichon & $1(0.16)$ & $6533(1.48)$ & 0.17 & $0.02-1.21$ & 0.076 & \\
\hline & Chihuahua & $1(0.16)$ & $11,709(2.64)$ & 0.09 & $0.01-0.67$ & 0.019 & \\
\hline & Border Terrier & $0(0.00)$ & $5379(1.21)$ & $\sim$ & $\sim$ & $\sim$ & \\
\hline \multirow[t]{8}{*}{$\begin{array}{l}\text { Kennel Club } \\
\text { Breed Group }\end{array}$} & $\begin{array}{l}\text { Breed not Kennel Club } \\
\text { recognised }\end{array}$ & $121(19.67)$ & $147,800(33.50)$ & Base & & & $<0.001$ \\
\hline & Toy & $31(5.04)$ & 56,669 (12.84) & 0.67 & $0.45-0.99$ & 0.045 & \\
\hline & Utility & $33(5.37)$ & 44,511 (10.09) & 0.91 & $0.62-1.33$ & 0.614 & \\
\hline & Terrier & 49 (7.97) & $57,913(13.13)$ & 1.03 & $0.74-1.44$ & 0.846 & \\
\hline & Gundog & $256(41.63)$ & $69,021(15.64)$ & 4.53 & $3.65-5.63$ & $<0.001$ & \\
\hline & Hound & $14(2.28)$ & $15,556(3.53)$ & 1.10 & $0.63-1.91$ & 0.737 & \\
\hline & Pastoral & $65(10.57)$ & $28,414(6.44)$ & 2.79 & $2.07-3.78$ & $<0.001$ & \\
\hline & Working & $46(7.48)$ & $21,353(4.84)$ & 2.63 & $1.87-3.70$ & $<0.001$ & \\
\hline \multirow{6}{*}{$\begin{array}{l}\text { Adult (> } 18 \text { months) } \\
\text { bodyweight (kg) }\end{array}$} & $<10.0$ & $36(5.84)$ & $98,298(22.20)$ & Base & & & $<0.001$ \\
\hline & $10.0-<20.0$ & 89 (14.45) & $89,741(20.27)$ & 2.71 & $1.84-3.99$ & $<0.001$ & \\
\hline & $20.0-<30.0$ & $115(18.67)$ & $65,740(14.85)$ & 4.78 & $3.28-6.95$ & $<0.001$ & \\
\hline & $30.0-<40.0$ & $187(30.36)$ & $43,560(9.84)$ & 11.72 & $8.20-16.75$ & $<0.001$ & \\
\hline & $\geq 40.0$ & $147(23.86)$ & $23,153(5.23)$ & 17.34 & $12.04-24.97$ & $<0.001$ & \\
\hline & Unavailable & $42(6.82)$ & $122,247(27.61)$ & 0.94 & $0.60-1.46$ & 0.779 & \\
\hline \multirow{2}{*}{$\begin{array}{l}\text { Bodyweight relative } \\
\text { to breed mean }\end{array}$} & Lower & $200(32.47)$ & $179,134(40.46)$ & Base & & & $<0.001$ \\
\hline & Equal/Higher & $374(60.71)$ & $140,861(31.82)$ & 2.38 & $2.00-2.82$ & $<0.001$ & \\
\hline
\end{tabular}


Table 1 Descriptive and univariable logistic regression results for risk factors associated with incident diagnoses during 2013 of elbow joint disease in dogs under primary veterinary care in the VetCompass ${ }^{\mathrm{TM}}$ Programme in the UK. Column percentages shown in brackets. ${ }^{*} \mathrm{Cl}$ confidence interval (Continued)

\begin{tabular}{|c|c|c|c|c|c|c|c|}
\hline Variable & Category & Case No. (\%) & $\begin{array}{l}\text { Non-case No. } \\
(\%)\end{array}$ & $\begin{array}{l}\text { Odds } \\
\text { ratio }\end{array}$ & $95 \% \mathrm{Cl}^{*}$ & $\begin{array}{l}\text { Category } \\
P \text {-value }\end{array}$ & $\begin{array}{l}\text { Variable } \\
P \text {-value }\end{array}$ \\
\hline & Unavailable & $42(6.82)$ & $122,744(27.72)$ & 0.31 & $0.22-0.43$ & $<0.001$ & \\
\hline \multirow[t]{6}{*}{ Age (years) } & $<3.0$ years & $108(17.53)$ & $173,638(39.22)$ & Base & & & $<0.001$ \\
\hline & $3.0-<6.0$ years & 103 (16.72) & $112,371(25.38)$ & 1.47 & $1.12-1.93$ & 0.005 & \\
\hline & $6.0-<9.0$ years & $142(23.05)$ & $74,082(16.73)$ & 3.08 & $2.40-3.96$ & $<0.001$ & \\
\hline & $9.0-<12.0$ years & 149 (24.19) & $44,891(10.14)$ & 5.34 & $4.16-6.84$ & $<0.001$ & \\
\hline & $\geq 12.0$ years & $112(18.18)$ & $31,666(7.15)$ & 5.69 & $4.36-7.41$ & $<0.001$ & \\
\hline & Unavailable & $2(0.32)$ & 6091 (1.38) & 0.53 & $0.13-2.14$ & 0.371 & \\
\hline \multirow[t]{3}{*}{ Sex } & Female & 244 (39.61) & $213,489(48.22)$ & Base & & & $<0.001$ \\
\hline & Male & $372(60.39)$ & $226,969(51.26)$ & 1.43 & $1.22-1.69$ & $<0.001$ & \\
\hline & Unavailable & $0(0.00)$ & $2281(0.52)$ & $\sim$ & $\sim$ & $\sim$ & \\
\hline \multirow[t]{3}{*}{ Neuter } & Entire & $122(19.81)$ & $174,753(39.47)$ & Base & & & $<0.001$ \\
\hline & Neutered & $360(58.44)$ & $198,298(44.79)$ & 2.60 & $2.12-3.19$ & $<0.001$ & \\
\hline & Unavailable & $134(21.75)$ & $69,688(15.74)$ & 2.75 & $2.15-3.52$ & $<0.001$ & \\
\hline \multirow[t]{3}{*}{ Insurance } & Non-insured & $27,320(6.17)$ & $41(6.66)$ & Base & & & $<0.001$ \\
\hline & Insured & $54,136(12.23)$ & $182(29.55)$ & 2.24 & $1.60-3.14$ & $<0.001$ & \\
\hline & Unavailable & $361,283(81.60)$ & $393(63.80)$ & 0.72 & $0.53-1.00$ & 0.050 & \\
\hline
\end{tabular}

Of the non-case dogs with data available on the variable, 331,113 (75.04\%) were purebred, 213,489 (48.47\%) were female, 198,298 (53.16\%) were neutered and 54,136 (66.46\%) were insured. The median adult bodyweight for non-cases was $16.20 \mathrm{~kg}$ (IQR: $8.90-27.50$, range 0.3099.95) and the median age was 4.06 years (IQR: $1.69-$ 7.52 , range $0.00-24.68)$. The most common breeds among the non-case dogs were Staffordshire Bull Terrier (31,897, 7.20\%), Labrador Retriever (30,894, 6.98\%), Jack Russell Terrier $(27,407,6.19 \%)$ and Cocker Spaniel (15, $458,3.49 \%$ ) accompanied by a substantial population of crossbred dogs $(110,124,24.87 \%)$ (Table 1). Data completeness varied between the variables assessed: breed $99.66 \%$, age $98.65 \%$, sex $99.50 \%$, bodyweight at any age $88.94 \%$, insurance $18.78 \%$, and neuter $84.12 \%$. There were $376(61.04 \%)$ cases recorded as bilateral with 123 (19.97\%) affected only on the left side and 117 (18.99\%) affected only on the right side.

\section{Clinical care}

Of the 616 incident cases, the following proportions of specific types of elbow joint disease were recorded at any date in the clinical records: osteoarthritis (or degenerative joint disease) ( $n=468,75.97 \%)$, elbow dysplasia (190, $30.84 \%)$, traumatic $(41,6.66 \%)$, septic arthritis $(11,1.79 \%)$, immune mediated $(7,1.14 \%)$, polyarthritis $(3,0.49 \%)$ and neoplastic $(3,0.49 \%)$. The most common presenting signs recorded as described by the owners were lameness $(n=$
$466,75.65 \%)$, difficulty exercising $(123,19.97 \%)$, pain $(86$, $13.96 \%)$ and inflammation (4, 0.65\%).

The elbow joint disease was noted as an incidental finding during a clinical examination for presentation for another reason in $63(10.23 \%)$ cases. The most common findings recorded on veterinary examination were pain $(n=283$, $45.94 \%)$, lameness $(278,45.13 \%)$, reduced range of movement (243, 39.45\%), crepitus $(162,26.30 \%)$ and inflammation or joint thickening $(133,21.59 \%)$. The diagnostic process included the following aids: radiography $(n=330,53.57 \%)$, computerized tomography (37, 6.01\%), arthroscopy (20, $3.26 \%$ ) and magnetic resonance imaging [MRI] (2, 0.33\%). Among 330 dogs that had at least one of these four diagnostic procedures, the most common specific diagnoses recorded were osteoarthritis $(n=101,30.61 \%)$ and coronoid disease (81, 24.55\%) (Table 2). Overall, 153 (24.84\%) of the elbow joint cases were referred for advanced veterinary care.

At least one medication was prescribed or used on 573 (93.02\%) cases. Common medications used included nonsteroidal anti-inflammatory drugs [NSAIDS] $(n=544$, $88.31 \%)$, tramadol $(121,19.64 \%)$, disease modifying agents $(118,19.16 \%)$, systemic glucocorticoids $(19,3.08 \%)$, gabapentin $(11,1.79 \%)$, intra-articular medications $(5,0.81 \%)$, amantadine $(3,0.49 \%)$. Nutraceuticals were used or recommended in $248(40.26 \%)$ of cases. Therapeutic surgery was performed on 85 (13.80\%) cases.

Of 167 incident cases that had died by the end of the study, the median age at death was 12.09 years (IQR 9.69-13.90, range 0.94-17.10). Of 153 dogs with 
Table 2 Specific veterinary diagnoses recorded in dogs under primary veterinary care in the VetCompass ${ }^{\mathrm{TM}}$ Programme in the UK with elbow joint disease that had at least one of the following: radiography, computerized tomography [CT], arthroscopy and magnetic resonance imaging [MRI]. $N=330$

\begin{tabular}{lll}
\hline Diagnosis & Frequency & Percent \\
\hline Osteoarthritis & 101 & 30.61 \\
Coronoid disease & 81 & 24.55 \\
Inconclusive & 54 & 16.36 \\
Fracture & 26 & 7.88 \\
Elbow incongruity & 25 & 7.58 \\
Osteochondritis dissecans & 19 & 5.76 \\
Ununited anconeal process & 11 & 3.33 \\
Incomplete ossification of the humeral condyle & 7 & 2.12 \\
Joint mouse & 2 & 0.61 \\
Neoplasia & 2 & 0.61 \\
Congenital elbow luxation & 1 & 0.30 \\
Polyarthritic elbow joint disease & 1 & 0.30 \\
Total & 330 & \\
\hline
\end{tabular}

information recorded, 146 (95.42\%) deaths involved euthanasia while 7 (4.58\%) were unassisted. Of 109 euthanasia cases with information available, elbow joint disease contributed to the decision to euthanase in $45(41.28 \%)$ dogs.

\section{Risk factors}

All tested variables were liberally associated with elbow joint disease in univariable logistic regression modelling and were evaluated using multivariable logistic regression modelling as described in the methods (Table 1). The final main breed-focused multivariable model retained six risk factors: breed, bodyweight relative to breed-sex mean, age, sex, neutered and insurance (Table 3). No biologically significant interactions were identified. The final model was improved by inclusion of the clinic attended as a random effect (rho: 0.03 indicating that $3 \%$ of the variability was accounted for by the clinic attended, $P<0.001$ ) and these results were reported. The final unclustered model showed acceptable model-fit (Hosmer-Lemeshow test statistic: $P=0.089$ ) and good discrimination (area under the ROC curve: 0.829 ).

After accounting for the effects of the other variables evaluated, five breeds showed increased odds of elbow joint disease compared with crossbred dogs: Rottweiler (OR: 6.16, 95\% CI 3.89-9.75, $P<0.001$ ), Labrador Retriever (OR: 5.94, 95\% CI 4.65-7.60, $P<0.001$ ), German Shepherd Dog (OR: 4.13, 95\% CI 2.88-5.93, $P<0.001$ ), Golden Retriever (OR: 3.11, 95\% CI 1.93-5.00, $P<0.001$ ) and English Springer Spaniel (OR: 2.00, 95\% CI 1.26-3.18, $P=0.003)$. Two breeds showed reduced odds of elbow joint disease compared with crossbreds: Jack Russell
Terrier (OR: 0.38, 95\% CI 0.20-0.72, $P=0.003$ ) and West Highland White Terrier (OR: 0.27, 95\% CI 0.10-0.73, $P=$ $0.010)$. Individual dogs with an adult bodyweight that was equal or higher than their breed/sex mean had 2.00 (95\% CI 1.68-2.39, $P<0.001$ ) times the odds of elbow joint disease compared with dogs that weighed below their breed/ sex mean. Ageing was associated with increasing odds of elbow joint disease. Compared with dogs aged $<3.0$ years, dogs aged $9.0-<12.0$ years had 2.56 times the odds $(95 \%$ CI 1.97-3.32, $P<0.001$ ) of elbow joint disease. Males had 1.47 times the odds (95\% CI 1.25-1.73, $P<0.001$ ) of elbow joint disease compared with females. Neutered animals had 1.69 times the odds (95\% CI 1.37-2.10, $P<0.001)$ of elbow joint disease compared with entire animals. Insured dogs had 2.32 (95\% CI 1.64-3.29, $P<0.001)$ times the odds of elbow joint disease compared with uninsured dogs (Table 3).

As described in the methods, purebred and Kennel Club breed group individually replaced the breed variable in the final breed-focused multivariable model while adult bodyweight replaced breed and bodyweight relative to $\mathrm{breed} / \mathrm{sex}$ mean. Purebred dogs had 1.70 times the odds (95\% CI 1.37-2.10, $P<0.001$ ) compared with crossbred dogs. Three of the seven Kennel Club breed groups showed higher odds of elbow joint disease compared with dogs of breeds that are not recognized by the Kennel Club: Gundog (OR: 3.94, 95\% CI 3.17-4.90, $P<0.001$ ), Working (OR: 3.00, 95\% CI 2.13-4.23, $P<0.001$ ) and Pastoral (OR: 2.54, 95\% CI 1.87-3.43, $P<0.001)$. The odds of elbow joint disease increased substantially as adult bodyweight increased. Dogs weighing $30.0-<40.0 \mathrm{~kg}$ had 9.84 times the odds $(95 \% \mathrm{CI}$ : 6.87-14.08, $\mathrm{P}<0.001$ ) (Table 4).

\section{Discussion}

To date, this is the largest primary-care veterinary study to provide epidemiological data on elbow joint disease in dogs and revealed a significant burden of elbow osteoarthritis in the wider dog population. Reliable prevalence data that is relevant to primary care is needed to help inform and focus health reforms in dogs, particularly those associated with breed characteristics $[46,47]$. A prevalence of $0.56 \%$ was shown from a population of 455,069 dogs from 304 clinics, indicating elbow joint disease is not as prevalent as patellar luxation [48] but had a similar prevalence to cruciate ligament rupture which is considered a significant health and financial burden [49]. Although previous estimates of breed prevalence for elbow dysplasia has varied from 0.01 to 0.89 [50], these populations are usually based on screening programmes and therefore do not represent the wider true dog population. Studies based on data derived from international schemes for assessing hip and elbow dyplasia are often biased through positive selection of dogs with 
Table 3 Final breed-focused mixed effects multivariable logistic regression model for risk factors associated with diagnosis of elbow joint disease in dogs under primary veterinary care in the VetCompass ${ }^{\mathrm{TM}}$ Programme in the UK. ${ }^{*} \mathrm{Cl}$ confidence interval

\begin{tabular}{|c|c|c|c|c|}
\hline Variable & Category & Odds ratio & $95 \% \mathrm{Cl}^{*}$ & $P$-value \\
\hline \multirow[t]{23}{*}{ Breed } & Crossbreed & 1.00 & & \\
\hline & Rottweiler & 6.16 & $3.89-9.75$ & $<0.001$ \\
\hline & Labrador Retriever & 5.94 & $4.65-7.60$ & $<0.001$ \\
\hline & German Shepherd Dog & 4.13 & $2.88-5.93$ & $<0.001$ \\
\hline & Golden Retriever & 3.11 & $1.93-5.00$ & $<0.001$ \\
\hline & English Springer Spaniel & 2.00 & $1.26-3.18$ & 0.003 \\
\hline & Other purebreds & 1.39 & $1.05-1.84$ & 0.021 \\
\hline & Lhasa Apso & 1.37 & $0.66-2.82$ & 0.396 \\
\hline & Unknown & 1.36 & $0.19-9.79$ & 0.761 \\
\hline & Staffordshire Bull Terrier & 1.27 & $0.85-1.89$ & 0.243 \\
\hline & Cavalier King Charles Spaniel & 1.15 & $0.62-2.15$ & 0.655 \\
\hline & Border Collie & 1.04 & $0.58-1.85$ & 0.900 \\
\hline & Cocker Spaniel & 0.95 & $0.54-1.67$ & 0.859 \\
\hline & Yorkshire Terrier & 0.74 & $0.40-1.39$ & 0.353 \\
\hline & Boxer & 0.68 & $0.25-1.85$ & 0.452 \\
\hline & Pug & 0.66 & $0.16-2.67$ & 0.557 \\
\hline & Shih-tzu & 0.46 & $0.19-1.14$ & 0.095 \\
\hline & Husky & 0.41 & $0.06-2.96$ & 0.379 \\
\hline & Jack Russell Terrier & 0.38 & $0.20-0.72$ & 0.003 \\
\hline & West Highland White Terrier & 0.27 & $0.10-0.73$ & 0.010 \\
\hline & Bichon & 0.18 & $0.03-1.30$ & 0.090 \\
\hline & Chihuahua & 0.16 & $0.02-1.18$ & 0.072 \\
\hline & Border Terrier & 0.00 & $\sim$ & 0.991 \\
\hline \multirow{3}{*}{$\begin{array}{l}\text { Bodyweight relative } \\
\text { to breed/sex mean }\end{array}$} & Lower & 1.00 & & \\
\hline & Equal/Higher & 2.00 & $1.68-2.39$ & $<0.001$ \\
\hline & Unavailable & 0.48 & $0.34-0.67$ & $<0.001$ \\
\hline \multirow[t]{6}{*}{ Age (years) } & $<3.0$ & 1.00 & & \\
\hline & $3.0-<6.0$ years & 0.84 & $0.64-1.11$ & 0.223 \\
\hline & $6.0-<9.0$ years & 1.47 & $1.13-1.92$ & 0.004 \\
\hline & $9.0-<12.0$ years & 2.56 & $1.97-3.32$ & $<0.001$ \\
\hline & $>$ or $=12.0$ years & 3.70 & $2.81-4.87$ & $<0.001$ \\
\hline & Unavailable & 0.80 & $0.20-3.26$ & 0.758 \\
\hline \multirow[t]{3}{*}{ Sex } & Female & 1.00 & & \\
\hline & Male & 1.47 & $1.25-1.73$ & $<0.001$ \\
\hline & Unavailable & 0.00 & $\sim$ & 0.995 \\
\hline \multirow[t]{3}{*}{ Neutered } & Entire & 1.00 & & \\
\hline & Neutered & 1.69 & $1.37-2.10$ & $<0.001$ \\
\hline & Unavailable & 1.54 & $1.16-2.05$ & 0.003 \\
\hline \multirow[t]{3}{*}{ Insurance } & Uninsured & 1.00 & & \\
\hline & Insured & 2.32 & $1.64-3.29$ & $<0.001$ \\
\hline & Unavailable & 1.17 & $0.82-1.67$ & 0.381 \\
\hline
\end{tabular}


Table 4 Results for Purebred status and Kennel Club Breed Group after replacing the breed variable in the final breed-focused mixed effects multivariable logistic regression model (along with age, bodyweight relative to breed mean, sex, neutered and insurance status) and for Adult (> 18 months) bodyweight (kg) that replaced the breed and bodyweight relative to breed mean variables in the final breed-focused mixed effects multivariable logistic regression model (with age, sex, neutered and insurance status). These results report associations between these risk factors and a diagnosis of elbow joint disease in dogs under primary veterinary care in the VetCompass ${ }^{\mathrm{TM}}$ Programme in the UK. ${ }^{*} \mathrm{Cl}$ confidence interval

\begin{tabular}{|c|c|c|c|c|}
\hline Variable & Category & Odds ratio & $95 \% \mathrm{Cl}^{*}$ & Category $P$-value \\
\hline \multirow[t]{2}{*}{ Purebred status } & Crossbred & 1.00 & & \\
\hline & Purebred & 1.70 & $1.37-2.10$ & $<0.001$ \\
\hline \multirow[t]{8}{*}{ Kennel Club Breed Group } & Breed not KC-recognised & 1.00 & & \\
\hline & Gundog & 3.94 & $3.17-4.90$ & $<0.001$ \\
\hline & Working & 3.00 & $2.13-4.23$ & $<0.001$ \\
\hline & Pastoral & 2.54 & $1.87-3.43$ & $<0.001$ \\
\hline & Hound & 1.06 & $0.61-1.84$ & 0.843 \\
\hline & Utility & 1.04 & $0.71-1.53$ & 0.851 \\
\hline & Terrier & 0.98 & $0.70-1.37$ & 0.921 \\
\hline & Toy & 0.76 & $0.51-1.12$ & 0.164 \\
\hline \multirow[t]{6}{*}{ Adult (> 18 months) bodyweight (kg) } & $<10.0$ & 1.00 & & \\
\hline & $10.0-<20.0$ & 2.35 & $1.59-3.46$ & $<0.001$ \\
\hline & $20.0-<30.0$ & 4.10 & $2.81-5.96$ & $<0.001$ \\
\hline & $30.0-<40.0$ & 9.84 & $6.87-14.08$ & $<0.001$ \\
\hline & $\geq 40.0$ & 15.03 & $10.39-21.74$ & $<0.001$ \\
\hline & Unavailable & 1.33 & $0.84-2.10$ & 0.218 \\
\hline
\end{tabular}

phenotypically 'good' joints [51], the voluntary nature of these schemes, the relatively low uptake across the total population of dogs, and the inclusion of predisposed phenotypes. Even after 40 years of enrolment in some programmes, there has been only minor changes reported in elbow dysplasia disease prevalence [52]. The application of anonymised primary care veterinary data which have not been 'vetted' in advance of inclusion in analyses and that are not biased to breeds which are known to have elbow dysplasia (and hence subject to screening) are therefore more likely to capture a representative estimate of disease for the entire population of dogs. Of course, these data rely heavily on the accuracy and completeness of clinical records which can be affected by differing diagnostic options and variable data recording of a range of clinical conditions.

The current study substantiated some previously reported breed-related variation in prevalence of elbow disease. The breeds with the highest prevalence were mainly large breeds and included Labrador Retriever, Rottweiler, Golden Retriever, German Shepherd Dogs, and English Springer Spaniels. Additional breeds with high prevalence of elbow dysplasia based on data from screening programmes, but not identified from the current primary care study included the Chow Chow, Bernese Mountain Dog and Newfoundland [50]. Male and neutered dogs were also more prevalent in the elbow disease group, with around $60 \%$ being male and $75 \%$ neutered. A predominance of male dogs has been reported previously for medial coronoid disease (the commonest form of elbow dysplasia) [1], and the male to female ratio for elbow dysplasia in Labradors and Golden Retrievers has been shown to be 2.2:1 [21, 22, 53]. The predisposition of male dogs could be due to dominant inheritance with reduced penetrance in female dogs, or associated factors such as neutering, exercise levels, growth rates and overall weight [54].

Purebred dogs had 1.7 times the odds of diagnosis of elbow disease, and the breeds at greatest risk of elbow disease included some of those previously identified as at risk of osteoarthritis and elbow dysplasia, in addition to English Springer Spaniels. The breeds with the highest risk of elbow disease were Labrador Retriever, Rottweiler, Golden Retriever, and German Shepherd, and these breeds have also previously demonstrated increased risk for osteoarthritis development [30]. Therefore, the elbow may be a significant contributing joint to the overall levels of osteoarthritis seen in the dog population. The breed-specific risk and increased prevalence in purebreds are suggestive of a genetic component to elbow disease and this is corroborated by heritability studies. Labrador Retriever heritability in the UK for elbow dysplasia was reported at 0.19 based upon 3613 elbow scores [25]. German Rottweilers have been 
reported with heritability of 0.28 [22], and Swedish Rottweilers at 0.34 [24]. German Shepherds have a heritability for elbow dysplasia of 0.6 [55], and 0.45 for Golden Retrievers [53]. The identification of increased risk in English Springer Spaniels in the current study may be due to their breed association with humeral intracondylar fissures (HIFs), which can predispose to lameness and low energy fractures $[5,6]$. In a prospective observational study of English Springer Spaniels without a history of lameness, CT scans revealed HIF in $14 \%$ of the dogs, and around $50 \%$ had medial coronoid process disease changes, with a total of $60 \%$ of apparently clinically normal dogs showing osteoarthritic change [56]. Data from the subset of dogs having diagnostic imaging in this study identified IOHC/HIF in $2 \%$ of these dogs. It is interesting that the groups of dogs bred for working, including Kennel Club Gundog, Pastoral and Working groups, all had significantly increased odds ratios for elbow disease. Whether elbow disease manifests more in these dogs due to their breed intended growth rates, sizes or elbow conformation remains unclear at this stage.

This study also identified breeds protected for elbow disease, including Jack Russell Terriers and West Highland White Terriers. These were not the same breeds as previously reported at reduced risk in the most up-todate long-term analysis of screening programme data which included 500 dogs. That study identified Boxer, Flat coated retriever, Bichon, Cavalier King Charles spaniel and Briard as low risk for elbow dysplasia [50]. The current study identified Boxers with an odds ratio of 0.68, although Cavalier King Charles spaniels had an odds ratio of 1.15. This difference could relate to the current study being more inclusive of elbow disease in general, or simply because it represents a bigger sample population which is not biased by owner decisionmaking on which dogs are submitted for radiographic survey.

In this current large population study, $61 \%$ of cases were diagnosed with bilateral elbow disease. The current study covered all types of elbow disease, whereas most prior information determining rates of bilateralism comes from elbow dysplasia studies with $25-80 \%$ of dogs reported with bilateral disease $[8,57,58]$. In any case, the high level of bilateral disease and functional impairment suggests that elbow joint disease is a significant welfare problem for affected individuals.

The differences identified here between owner perception and veterinary assessments are worth considering in terms of welfare and public education. Owners predominantly focussed on externally visible clinical signs such as lameness $(75.65 \%)$ or problems exercising (19.97\%), with only a smaller proportion reporting internal affective issues such as pain $(13.96 \%)$ as a presenting complaint. Conversely, veterinary assessment recorded pain $(45.94 \%)$ as the most common clinical finding. Dog owners have previously been shown to be poor at predicting and interpretting behavioural adaptation to pain [59], and therefore reliance on dog owners to identify chronic pain may be unsafe [60]. Additionally, $10.23 \%$ of elbow joint disease cases were identified incidentally at routine veterinary appointments which suggests that many owners may normalise these clinical signs as typical of aging. Veterinary examination can reveal and detect aspects of joint disease that are not appreciable to untrained owners and it may be unfair to assume that all elbow joint cases should be easily recognisable to owners. Typical clinical signs indicative of advanced joint remodelling associated with osteoarthritis were identified on veterinary examination have been reported in $1 / 5$ (thickening) to $1 / 4$ (crepitus) dogs, and $50 \%$ of diagnoses were made on clinical examination [61].

Consistent with the predominance of older dogs identified with elbow joint disease in the current study, osteoarthritis (degenerative joint disease) was diagnosed in over $75 \%$ of cases. As discussed above, these later stage presentations are most likely secondary to preexisting elbow dysplasia but could also result from some forms of primary osteoarthritis [16]. Studies show that both conservative management [62] and surgical treatment of elbow dysplasia ultimately lead to osteoarthritis $[63,64]$. The protracted time lag from inciting cause to clinical presentation for chronic diseases such as osteoarthritis combined with the data-availability constraints of our type of epidemiological study did not permit fuller exploration of the natural history of these cases. It does however clearly highlight that, by whatever the route, elbow osteoarthritis is the most common disease present in the elbows of dogs under primary care, with its substantial impact on pain, mobility and welfare. From a welfare perspective, it is further notable that their elbow joint disease, which was mostly diagnosed as osteoarthritis, contributed to the decision to euthanase in $41.28 \%$ of the cases euthanased during the study, indicating another significant welfare impact. An exploration of summary welfare impact among common disorders of dogs in the UK identified osteoarthritis with the second highest overall welfare impact score among the eight disorders evaluated [65]. The current study also provides the first indication of a population incidence for rare elbow joint diseases such as septic or immune mediated polyarthritis, both being under $2 \%$, and corroborates the limited literature from a handful of small case series from referral populations [2, 66-68].

A tendency towards increased diagnosis in insured dogs was shown here and has also been demonstrated for other orthopaedic conditions [30, 48, 69]. Reduced financial restrictions or potentially differing client/owner 
expectations when insured may underlie this recurring phenomenon. Interestingly, the influence of insurance on diagnostic outcomes appears to vary by condition, being highest for cruciate ligament rupture $(4 \mathrm{x})$, then elbow disease (2.35x) and then patellar luxation (1.9x). This perhaps reflects the differential confidence by primary-care practitioners in diagnosis-making between these conditions and the subsequent need for additional costly diagnostics to assess the more uncertain diagnoses. In the current study, only around half of cases included diagnostic imaging within diagnostic protocols used, suggesting a relatively high level of clinical confidence in these diagnoses being made in primary veterinary care. Plain radiography dominated among the diagnoses that included diagnostic imaging, perhaps due to its ease of use and fairly universal access in primary care. However, plain radiography is associated with a relatively high proportion of false-negative diagnoses (10-69\%) in large groups of dogs with elbow related lameness [70, 71]. Hence, advanced imaging is frequently recommended but often requires referral to ensure access to this diagnostic modality. Direct diagnosis of common forms of elbow dysplasia (coronoid disease, elbow incongruity, osteochondritis dissecans and ununited anconeal process) accounted for $41 \%$ of diagnoses, with medial coronoid process disease accounting for 59\% of elbow dysplasia types. This is lower than reported in screening programme populations [72] with medial coronoid disease being present in $96 \%$ of elbow dysplasia dogs, perhaps because the use of plain radiography significantly reduces accurate diagnosis $[70,71]$.

Despite many years of research, the exact aetiology of elbow dysplasia remains unclear. Several overarching theories have been described including osteochondrosis [73], differing types of elbow incongruity [54], and muscular biomechanical force mismatch [74]. Whatever the aetiology, these disease entities are considered to be the result of the interplay of genetics and environment such as high energy diets driving rapid growth or excessive exercise [4]. Although elbow dysplasia and joint incongruity have been well described as a driver of joint arthrosis in young dogs $[1,12,75]$, osteoarthritis development in absence of a primary alternative joint disease, so called primary osteoarthritis, is thought to be rare [11].

Being above average bodyweight for the breed and sex was identified as a significant risk factor for elbow disease. Experimental dog colonies have clearly demonstrated that increased calorie intake and hence increased bodyweight positively are associated with increased levels of osteoarthritis. In a longitudinal study following seven litters of Labrador Retrievers where one group was fed ad-lib and the other $25 \%$ fewer calories, the reducedcalorie group had $26 \%$ lower mean body mass. The adlib fed dogs showed significantly greater radiographic severity of osteoarthritic change at 6 years of age, although histopathology did not any differences at end of life. Overall, by 6 years of age, radiographic osteoarthritis was seen in $19.1 \%$ of dogs [16]. Age was significantly associated with prevalence and severity of osteoarthritis in the Labrador Retriever colony dogs, which was also demonstrated as a risk factor for incidence in the current study. Elbow dysplasia has historically been considered one of the main causes of elbow osteoarthritis $[1,11,12,75]$, however, rather unexpectedly, none of the dogs in the colony had any indication of a pre-existing disease such as elbow dysplasia based on the radiographic and histological signs of the presence of a fragmented medial coronoid process (FMCP), un-united anconeal process (UAP) and osteochondrosis or osteochondritis dissecans (OCD). For the first time, elbow osteoarthritis was documented as a potentially primary osteoarthritis. This suggests that some of the dogs identified in the current study with elbow disease may have had primary osteoarthritis.

The clinical management of elbow joint disease in the current study, dominated by osteoarthritis, included at least one medication in most dogs. Medication included a predominance of NSAIDs (88\%) and also a relatively high level of tramadol usage (19\%). Tramadol for osteoarthritis management has been popularised in recent years following concerns over side-effects from NSAIDs [76]. However a recent randomised placebo controlled, cross-over, double blinded study irrefutably demonstrated that tramadol was only as effective as placebo and was significantly inferior to NSAIDs [77]. Interestingly, when compared to management of osteoarthritis overall in dogs, medical treatment is prescribed more frequently for the elbow than for osteoarthritis in general (93\% vs $75 \%$ respectively) [30]. This may reflect more debilitating effects perceived for osteoarthritis in the elbow compared with some other joints. Nutraceuticals were commonly used in primary-care practice, with $40 \%$ of dogs receiving them. Some elbow joint disease dogs were treated by surgical intervention in primary veterinary care $(14 \%)$, presumably for a primary disease such as medial coronoid process disease.

Two age related peaks in incidence were seen, corroborating the young and the old patterns previously described in referral populations [4]. In those referral populations, this pattern was attributed to primary elbow dysplasia driving lameness when young whereas the later peak has been attributed to the subsequent secondary osteoarthritis [1]. A risk factor for osteoarthritis is increasing age, notably above 8 years [30], and a similar peak was demonstrated for elbow disease with a peak in the 8-10 year range. When arthroscopic findings from 600 dogs with elbow lameness were reviewed, nearly $50 \%$ were in the younger age group 
peak (5-18 months of age), and $12 \%$ were in an old dog group ( $>6$ years). There was a notable difference in pathology with medial compartment erosions (deep ulcerations of the medial part of the joint with exposure of the subchondral bone - Modified Outerbridge Score 4) in $31 \%$ of old dogs and only $3 \%$ of young dogs. The Bernese Mountain dog was not seen in the older group, whereas mixed breeds were over-represented [78], suggestive perhaps of different disease entities occurring in the elbow of older vs young dogs, although in both groups medial coronoid disease predominated.

The limitations of using primary-care veterinary clinical data for research have been previously published $[27,33,79]$. The current study was limited by its retrospective nature and the use of clinical data that were not recorded primarily for research purposes and which therefore may have allowed some disease status misclassification. This study may have underrepresented elbow joint disease because true cases in the denominator population that were not presented for veterinary care during 2013 were not included as cases. It is also difficult to distinguish the natural history of elbow disease, as we can only presume elbow dysplasia to be a common underlying cause of the high level of elbow osteoarthritis. The bimodal incidence distribution, with a young dog peak in the first 2 years of life and a broader old dog peak fits with the literature descriptions of elbow dysplasia in the young leading to secondary osteoarthritis in the older dog. Furthermore, similar sex and breed distributions that are described for elbow dysplasia were also seen in the current study $[22,24,25,53$, 55]. Although the current study could not determine the proportion of osteoarthritis cases that had underlying elbow dysplasia, the study clearly highlights that, at any one time, there is a large population of dogs with elbow osteoarthritis in primary veterinary practice. This study also gives the most accurate measure of the prevalence of elbow disease in primary care dogs, and also provides, for the first time, an index of prevalence for rare conditions such as septic and immune mediated elbow joint disease. This study excluded dogs that were not under veterinary care and therefore may have introduced bias toward the increasingly neutered, insured and more closely monitored subset of the population that do receive veterinary care. Body condition scores were not available for this study and therefore analysis of association between obesity and elbow joint disease, although desirable, was not possible.

\section{Conclusions}

This is the largest epidemiological study based on primary care veterinary data to evaluate elbow joint disease in dogs and shows a prevalence of $0.56 \%$ in the UK. There were strong breed predispositions, in particular for large breed dogs, such as Labrador, Rottweiler, Golden Retriever and German Shepherd Dog, which align to breeds shown to be over-represented in elbow dysplasia studies. Notably, there was a very high level of bilateral disease at $61 \%$, and being male, neutered and weighing above the breed average were significant risk factors. Osteoarthritis was by far the most common specific cause for elbow joint disease. Tramadol is frequently used to manage the disease, although recent evidence worryingly suggests this is no more effective than placebo [80]. This study identified a significant welfare burden from elbow joint disease with over $40 \%$ of euthanasia cases during that period being attributed to their elbow joint disease. Based on the breed predisposition, high level of bilateral disease and impact on welfare, there is a significant case for improving breeding programmes and developing improved genetic assessment tools to reduce the burden of elbow joint disease.

\section{Abbreviations \\ Cl: Confidence interval; CT: Computerized tomography; EPR: Electronic patient record; FMCP: Fragmented medial coronoid process; HIF: Humeral intracondylar fissure; IEWG: The International Elbow Working Group; IQR: Interquartile range; KC : The Kennel Club; MRI: Magnetic resonance imaging; NSAIDS: Non-steroidal anti-inflammatory drugs; \\ OCD: Osteochondritis dissecans; OR: Odds ratio; UAP: Ununited anconeal process}

\section{Acknowledgements \\ Thanks to Noel Kennedy (RVC) for VetCompass ${ }^{\mathrm{TM}}$ software and programming development. We acknowledge the Medivet Veterinary Partnership, Vets4Pets/ Companion Care, Goddard Veterinary Group, Independent Vetcare, CVS Group, Linnaeus Group, Vets Now and the other UK practices who collaborate in VetCompass ${ }^{\mathrm{TM}}$. We are grateful to The Kennel Club and The Kennel Club Charitable Trust for supporting VetCompass ${ }^{\mathrm{TM}}$.}

\section{Authors' contributions}

$\mathrm{DON}, \mathrm{RH}$ and RM were responsible mainly for the conception and design, acquisition and extraction of data. DON carried out the analysis. DON, DB, $\mathrm{RH}, \mathrm{DC}$ and RM were involved in interpretation of the results, drafting and revising the manuscript and gave final approval of the version to be published. DON, DB, RH, DC and RM agree to be accountable for all aspects of the accuracy or integrity of the work.

\section{Funding}

DON was supported at the RVC for part of this study by an award from the Kennel Club Charitable Trust. Neither the Kennel Club Charitable Trust nor the Kennel Club had any input in the design of the study, the collection, analysis and interpretation of data or in writing the manuscript should be declared.

\section{Availability of data and materials}

The VetCompass dataset used for this study are available open access on the RVC data repository: http://researchonline.rvc.ac.uk/id/eprint/12408/ .

Ethics approval and consent to participate

Ethical approval was granted by the RVC Ethics and Welfare Committee (reference number SR2018-1652).

Consent for publication

Not applicable.

Competing interests

The authors have no conflicts of interest to declare. 


\section{Author details}

'Production and Population Health, The Royal Veterinary College, Hawkshead Lane, North Mymms, Hatfield, Herts AL9 7TA, UK. ${ }^{2}$ Clinical Sciences and Services, The Royal Veterinary College, Hawkshead Lane, North Mymms, Hatfield, Herts AL9 7TA, UK.

Received: 3 December 2019 Accepted: 5 February 2020

Published online: 14 February 2020

\section{References}

1. Demko J, McLaughlin R. Developmental orthopedic disease. Vet Clin Small Anim Pract. 2005;35(5):1111-35.

2. Mielke B, Comerford E, English K, Meeson R. Spontaneous septic arthritis of canine elbows: twenty-one cases. Vet Comp Orthopaed. 2018;31(06):488-93.

3. Morgan J, Wind A, Davidson A. Bone dysplasias in the labrador retriever: a radiographic study. J Am Anim Hosp Assoc. 1999;35(4):332-40.

4. Michelsen J. Canine elbow dysplasia: Aetiopathogenesis and current treatment recommendations. Vet J. 2013;196(1):12-9.

5. Moores AP, Moores AL. The natural history of humeral intracondylar fissure: an observational study of 30 dogs. J Small Anim Pract. 2017;58(6):337-41.

6. Marcellin-Little DJ, Deyoung DJ, Ferris KK, Berry CM. Incomplete ossification of the humeral condyle in spaniels. Vet Surg. 1994;23(6):475-87.

7. Tobias KM, Johnston SA. Veterinary surgery : small animal. Elsevier Saunders: St Lousi; 2012.

8. Fitzpatrick N, Smith TJ, Evans RB, Yeadon R. Radiographic and arthroscopic findings in the elbow joints of 263 dogs with medial coronoid disease. Vet Surg. 2009;38(2):213-23.

9. Corley E, Carlson WD. Radiographic, genetic, and pathologic aspects of elbow dysplasia. J Am Vet Med Assoc. 1965;147:1651-3.

10. The International Elbow Working Group. The International Elbow Working Group: IEWG; 2019 [Available from: http://www.vet-iewg.org/.

11. Keller GG, Kreeger JM, Mann FA, Lattimer JC. Correlation of radiographic, necropsy and histologic findings in 8 dogs with elbow dysplasia. Vet Radiol Ultrasound. 1997;38(4):272-6.

12. Snaps F, Balligand M, Saunders J, Park R, Dondelinger R. Comparison of radiography, magnetic resonance imaging, and surgical findings in dogs with elbow dysplasia. Am J Vet Res. 1997;58(12):1367-70.

13. Zontine WJ, Weitkamp RA, Lippincott CL. Redefined type of elbow dysplasia involving calcified flexor tendons attached to the medial humeral epicondyle in three dogs. J Am Vet Med Assoc. 1989;194(8):1082-5.

14. Boulay JP. Fragmented medial coronoid process of the ulna in the dog. Vet Clin Small Anim Pract. 1998;28(1):51-74.

15. Burton NJ, Comerford EJ, Bailey M, Pead MJ, Owen MR. Digital analysis of ulnar trochlear notch sclerosis in Labrador retrievers. J Small Anim Pract. 2007:48(4):220-4

16. Huck JL, Biery DN, Lawler DF, Gregor TP, Runge JJ, Evans RH, et al. A longitudinal study of the influence of lifetime food restriction on development of osteoarthritis in the canine elbow. Vet Surg. 2009;38(2): 192-8.

17. Rovesti GL, Biasibetti M, Schumacher A, Fabiani M. The use of the computed tomography in the diagnostic protocol of the elbow in the dog: 24 joints. Vet Comp Orthopaed. 2002;15(01):35-43.

18. Moores AP, Benigni L, Lamb CR. Computed tomography versus arthroscopy for detection of canine elbow dysplasia lesions. Vet Surg. 2008:37(4):390-8.

19. Bartlett PC, Van Buren JW, Neterer M, Zhou C. Disease surveillance and referral bias in the veterinary medical database. Prev Vet Med. 2010;94(3-4): 264-71.

20. Ubbink GJ, Hazewinkel HA, van de Broek J, Rothuizen J. Familial clustering and risk analysis for fragmented coronoid process and elbow joint incongruity in Bernese Mountain dogs in the Netherlands. Am J Vet Res. 1999:60(9):1082-7.

21. Meyer-Lindenberg A, Fehr M, Nolte I. Co-existence of ununited anconeal process and fragmented medial coronoid process of the ulna in the dog. Small Anim Pract. 2006;47(2):61-5

22. Beuing R, Mues C, Tellhelm B, Erhardt G. Prevalence and inheritance of canine elbow dysplasia in German Rottweiler. J Anim Breed Genet. 2000; 117(6):375-83.

23. Janutta V, Hamann H, Klein S, Tellhelm B, Distl O. Genetic analysis of three different classification protocols for the evaluation of elbow dysplasia in German shepherd dogs. J Small Anim Pract. 2006;47(2):75-82.
24. Malm S, Fikse WF, Danell B, Strandberg E. Genetic variation and genetic trends in hip and elbow dysplasia in Swedish Rottweiler and Bernese Mountain dog. J Anim Breed Genet. 2008;125(6):403-12.

25. Lewis TW, Ilska JJ, Blott SC, Woolliams JA. Genetic evaluation of elbow scores and the relationship with hip scores in UK Labrador retrievers. Vet J. 2011:189(2):227-33.

26. Stock KF, Klein S, Tellhelm B, Distl O. Genetic analyses of elbow and hip dysplasia in the German shepherd dog. J Anim Breed Genet. 2011;128(3): 219-29.

27. O'Neill DG, Church DB, McGreevy PD, Thomson PC, Brodbelt DC. Prevalence of disorders recorded in dogs attending primary-care veterinary practices in England. PLoS One. 2014;9(3):1-16.

28. McGreevy PD, Nicholas FW. Some practical solutions to welfare problems in dog breeding. Anim Welf. 1999:8:329-41.

29. Harris GL, Brodbelt D, Church D, Humm K, McGreevy PD, Thomson PC, et al. Epidemiology, clinical management, and outcomes of dogs involved in road traffic accidents in the United Kingdom (2009-2014). J Vet Emerg Crit Care. 2018;28(2):140-8

30. Anderson KL, O'Neill DG, Brodbelt DC, Church DB, Meeson RL, Sargan D, et al. Prevalence, duration and risk factors for appendicular osteoarthritis in a UK dog population under primary veterinary care. Sci Rep. 2018:8(1):5641.

31. O'Neill DG, O'Sullivan AM, Manson EA, Church DB, McGreevy PD, Boag AK et al. Canine dystocia in 50 UK first-opinion emergency care veterinary practices: clinical management and outcomes. Vet Rec. 2019;184:409.

32. Hall JL, Owen L, Riddell A, Church DB, Brodbelt DC, O'Neill DG. Urinary incontinence in male dogs under primary veterinary care in England: prevalence and risk factors. J Small Anim Pract. 2019;60(February):86-95.

33. O'Neill DG, Lee MM, Brodbelt DC, Church DB, Sanchez RF. Corneal ulcerative disease in dogs under primary veterinary care in England: epidemiology and clinical management. Canine Genet Epidemiol. 2017:4(1):5.

34. RCVS Knowledge.Clinical Audit Toolkit. RCVS Knowledge; 2016 [Available from: http://knowledge.rcvs.org.uk/evidence-based-veterinary-medicine/ ebvm-resources/tools-guidelines-and-checklists/clinical-audit-toolkit/.

35. Viner B. Using audit to improve clinical effectiveness. In Pract. 2009;31(5): 240-3.

36. VetCompass. VetCompass ${ }^{T M}$ Programme London: RVC Electronic Media Unit; 2019 [Available from: http://www.rvc.ac.uk/VetCOMPASS/.

37. The VeNom Coding Group. VeNom Veterinary Nomenclature: VeNom Coding Group; 2019 [Available from: http://venomcoding.org.

38. Pearce N. Classification of epidemiological study designs. Int J Epidemiol. 2012:41(2):393-7.

39. The Kennel Club. Breed Information Centre: The Kennel Club Limited; 2019 [Available from: http://www.thekennelclub.org.uk/services/public/breed/

40. Scott M, Flaherty D, Currall J. Statistics: how many? J Small Anim Pract. 2012; 53(7):372-6

41. O'Neill DG, Scudder C, Faire JM, Church DB, McGreevy PD, Thomson PC, et al. Epidemiology of hyperadrenocorticism among 210,824 dogs attending primary-care veterinary practices in the UK from 2009 to 2014. J Small Anim Pract. 2016:57(7):365-73.

42. Kirkwood BR, Sterne JAC. Essential medical statistics. 2nd ed. Oxford: Blackwell Science; 2003.

43. O'Neill DG, Riddell A, Church DB, Owen L, Brodbelt DC, Hall JL. Urinary incontinence in bitches under primary veterinary care in England: prevalence and risk factors. J Small Anim Pract. 2017:58(12):685-93.

44. Dohoo I, Martin W, Stryhn H. Veterinary epidemiologic research. 2nd ed. VER Inc: Charlottetown, Canada; 2009

45. Hosmer DW, Lemeshow S, Sturdivant RX. Applied logistic regression. 3rd ed. Hoboken: Wiley; 2013

46. Collins LM, Asher L, Summers J, McGreevy P. Getting priorities straight: risk assessment and decision-making in the improvement of inherited disorders in pedigree dogs. Vet J. 2011;189(2):147-54.

47. Asher L, Diesel G, Summers JF, McGreevy PD, Collins LM. Inherited defects in pedigree dogs. Part 1: disorders related to breed standards. Vet J. 2009; 182(3):402-11.

48. O'Neill DG, Meeson RL, Sheridan A, Church DB, Brodbelt DC. The epidemiology of patellar luxation in dogs attending primary-care veterinary practices in England. Canine Genet Epidemiol. 2016;3(1):1-12.

49. Taylor-Brown FE, Meeson RL, Brodbelt DC, Church DB, McGreevy PD, Thomson PC, et al. Epidemiology of cranial cruciate ligament disease diagnosis in dogs attending primary-care veterinary practices in England. Vet Surg. 2015;44(6):777-83. 
50. Oberbauer AM, Keller GG, Famula TR. Long-term genetic selection reduced prevalence of hip and elbow dysplasia in 60 dog breeds. PLoS One. 2017; 12(2):e0172918.

51. Verhoeven G, Fortrie R, Van Ryssen B, Coopman F. Worldwide screening for canine hip dysplasia: where are we now? Vet Surg. 2012;41(1):10-9.

52. Coopman F, Verhoeven G, Saunders J, Duchateau L. Bree Hv. Prevalence of hip dysplasia, elbow dysplasia and humeral head osteochondrosis in dog breeds in Belgium. Vet Rec. 2008;163(22):654-8.

53. Guthrie S, Pidduck HG. Heritability of elbow osteochondrosis within a closed population of dogs. J Small Anim Pract. 1990;31(2):93-6.

54. Alves-Pimenta S, Ginja MM, Colaço B. Role of elbow incongruity in canine elbow dysplasia: advances in diagnostics and biomechanics. Vet Comp Orthopaed. 2019;32(02):087-96

55. Hou Y, Wang Y, Lu X, Zhang X, Zhao Q, Todhunter RJ, et al. Monitoring hip and elbow dysplasia achieved modest genetic improvement of 74 dog breeds over 40 years in USA. PLoS One. 2013;8(10):e76390.

56. Moores A, Agthe P, Schaafsma I. Prevalence of incomplete ossification of the humeral condyle and other abnormalities of the elbow in English springer spaniels. Vet Comp Orthopaed. 2012;25(03):211-6.

57. Van Ryssen B, van Bree H. Arthroscopic findings in 100 dogs with elbow lameness. Vet Rec. 1997;140(14):360.

58. Perry KL, Li L. A retrospective study of the short-term complication rate following 750 elective elbow arthroscopies. Vet Comp Orthopaed. 2014; 27(01):68-73.

59. Lush J, ljichi C. A preliminary investigation into personality and pain in dogs. J Vet Behav. 2018;24:62-8.

60. Brown DC, Boston RC, Farrar JT. Comparison of force plate gait analysis and owner assessment of pain using the canine brief pain inventory in dogs with osteoarthritis. J Vet Intern Med. 2013;27(1):22-30.

61. Pettitt RA, German AJ. Investigation and management of canine osteoarthritis. In Pract. 2015;37(Suppl 1):1-8.

62. Huibregtse B, Johnson A, Muhlbauer M, Pijanowski G. The effect of treatment of fragmented coronoid process on the development of osteoarthritis of the elbow. J Am Anim Hosp Assoc. 1994;30:190-5.

63. Barthélémy NP, Griffon DJ, Ragetly GR, Carrera I, Schaeffer DJ. Short- and longterm outcomes after arthroscopic treatment of Young large breed dogs with medial compartment disease of the elbow. Vet Surg. 2014;43(8):935-43.

64. Meyer-Lindenberg A, Langhann A, Fehr M, Nolte I. Arthrotomy versus arthroscopy in the treatment of the fragmented medial coronoid process of the ulna (FCP) in 421 dogs. Vet Comp Orthopaed. 2003;16(04):204-10.

65. Summers JF, O'Neill DG, Church D, Collins L, Sargan D, Brodbelt DC. Healthrelated welfare prioritisation of canine disorders using electronic health records in primary care practice in the UK. BMC Vet Res. 2019;15(1):163.

66. Stull JW, Evason M, Carr AP, Waldner C. Canine immune-mediated polyarthritis: clinical and laboratory findings in 83 cases in western Canada (1991-2001). Can Vet J. 2008:49(12):1195.

67. Clements DN, Gear RNA, Tattersall J, Carmichael S, Bennett D. Type immune-mediated polyarthritis in dogs: 39 cases (1997-2002). J Am Vet Med Assoc. 2004;224(8):1323-7.

68. Colopy SA, Baker TA, Muir P. Efficacy of leflunomide for treatment of immune-mediated polyarthritis in dogs: 14 cases (2006-2008). J Am Vet Med Assoc. 2010;236(3):312-8.

69. Jerram RM, Walker AM. Cranial cruciate ligament injury in the dog: pathophysiology, diagnosis and treatment. N Z Vet J. 2003;51(4):149-58.

70. Rau F, Wigger A, Tellhelm B, Zwick M, Klumpp S, Neumann A, et al. Observer variability and sensitivity of radiographic diagnosis of canine medial coronoid disease. Tierarztl Prax Ausg K Klientiere Heimtiere. 2011 39(05):313-22

71. Villamonte-Chevalier A, van Bree $H$, Broeckx BJG, Dingemanse W, Soler M, Van Ryssen B, et al. Assessment of medial coronoid disease in 180 canine lame elbow joints: a sensitivity and specificity comparison of radiographic, computed tomographic and arthroscopic findings. BMC Vet Res. 2015;11(1):243.

72. Lavrijsen ICM, Heuven HCM, Voorhout G, Meij BP, Theyse LFH, Leegwater PAJ, et al. Phenotypic and genetic evaluation of elbow dysplasia in Dutch Labrador retrievers, Golden retrievers, and Bernese Mountain dogs. Vet J. 2012;193(2):486-92.

73. Olsson S. The early diagnosis of fragmented coronoid process and osteochondritis dissecans of the canine elbow joint. J Am Anim Hosp Assoc (USA). 1983:19(5):616-26.
74. Hulse D, Young B, Beale B, Kowaleski M, Vannini R. Relationship of the biceps-brachialis complex to the medial coronoid process of the canine ulna. Vet Comp Orthopaed. 2010;23(03):173-6.

75. Lang J, Busato A, Baumgartner D, Flückiger M, Weber UT. Comparison of two classification protocols in the evaluation of elbow dysplasia in the dog. J Small Anim Pract. 1998;39(4):169-74.

76. Belshaw Z, Asher L, Dean RS. The attitudes of owners and veterinary professionals in the United Kingdom to the risk of adverse events associated with using non-steroidal anti-inflammatory drugs (NSAIDs) to treat dogs with osteoarthritis. Prev Vet Med. 2016;131:121-6.

77. Budsberg SC, Torres BT, Kleine SA, Sandberg GS, Berjeski AK. Lack of effectiveness of tramadol hydrochloride for the treatment of pain and joint dysfunction in dogs with chronic osteoarthritis. J Am Vet Med Assoc. 2018; 252(4):427-32.

78. Vermote K, Bergenhuyzen A, Gielen I, van Bree H, Duchateau L, Van Ryssen B. Elbow lameness in dogs of six years and older: arthroscopic and imaging findings of medial coronoid disease in 51 dogs. Vet Comp Orthopaed. 2010; 23(1):43-50.

79. O'Neill D, Church D, McGreevy P, Thomson P, Brodbelt D. Approaches to canine health surveillance. Canine Genet Epidemiol. 2014;1(1):2.

80. Budsberg S, Torres B, Kleine S, Sandberg G, Berjeski A. Lack of Treatment Efficacy of Tramadol on Pain and Dysfunction of Chronic Osteoarthritic (OA) Joints in Dogs. Vet Comp Orthopaed. 2018;31(S 01):A003.

\section{Publisher's Note}

Springer Nature remains neutral with regard to jurisdictional claims in published maps and institutional affiliations.
Ready to submit your research? Choose BMC and benefit from:

- fast, convenient online submission

- thorough peer review by experienced researchers in your field

- rapid publication on acceptance

- support for research data, including large and complex data types

- gold Open Access which fosters wider collaboration and increased citations

- maximum visibility for your research: over $100 \mathrm{M}$ website views per year

At $\mathrm{BMC}$, research is always in progress.

Learn more biomedcentral.com/submissions 